\title{
Controlled and tunable polymer particles' production using a single microfluidic device
}

\author{
Benzion Amoyav ${ }^{1} \cdot$ Ofra Benny $^{1}$ (1)
}

Received: 26 December 2017 / Accepted: 19 April 2018 / Published online: 26 April 2018

(c) The Author(s) 2018

\begin{abstract}
Microfluidics technology offers a new platform to control liquids under flow in small volumes. The advantage of using smallscale reactions for droplet generation along with the capacity to control the preparation parameters, making microfluidic chips an attractive technology for optimizing encapsulation formulations. However, one of the drawback in this methodology is the ability to obtain a wide range of droplet sizes, from sub-micron to microns using a single chip design. In fact, typically, droplet chips are used for micron-dimension particles, while nanoparticles' synthesis requires complex chips design (i.e., microreactors and staggered herringbone micromixer). Here, we introduce the development of a highly tunable and controlled encapsulation technique, using two polymer compositions, for generating particles ranging from microns to nano-size using the same simple single microfluidic chip design. Poly(lactic-co-glycolic acid) (PLGA 50:50) or PLGA/polyethylene glycol polymeric particles were prepared with focused-flow chip, yielding monodisperse particle batches. We show that by varying flow rate, solvent, surfactant and polymer composition, we were able to optimize particles' size and decrease polydispersity index, using simple chip designs with no further related adjustments or costs. Utilizing this platform, which offers tight tuning of particle properties, could offer an important tool for formulation development and can potentially pave the way towards a better precision nanomedicine.
\end{abstract}

Keywords Microfluidics $\cdot$ Nanoparticles $\cdot$ Microparticles $\cdot$ Polymeric particles $\cdot$ Focused flow

\section{Introduction}

The synthesis of well-defined monodisperse polymeric drug particles is a central challenge in the development of drug delivery systems, especially for injectable products. In contrast to traditional batch encapsulation techniques (Khadka et al. 2014; Niu et al. 2017), the rising technology of microfluidics enables to control fluid flow at the microscopic scale (Squires 2005). Downscaling processes with a microfluidic device offer many advantages including the low cost and faster parallel analysis for formulation development using many various conditions in a single chip (Novotný and Foret 2017; Valencia et al. 2012).

The ability of microfluidic devices to manipulate microand nano-liter volumes of liquid and to control the mixing

Ofra Benny

ofrab@ekmd.huji.ac.il

1 Faculty of Medicine, The School of Pharmacy, The Institute for Drug Research, The Hebrew University, Campus Ein Karem, 91120 Jerusalem, Israel process serves as an excellent tool for generating reproducible micro- and nano-sized particles, characterized by a controlled and narrow size distribution or distinct shapes, compared with that gained by batch synthesis (Duncanson et al. 2012; Zhao 2013).

Polymeric carriers are being widely used pre-clinically and clinically for slow release of drugs and for targeted therapy, in cancer and other diseases (Jain et al. 2012; Parveen and Sahoo 2008). Over the years, many medically approved products are being launched, such as Eligard $\AA$, Neulasta ${ }$, Abraxane ${ }^{\circledR}$, Doxil ${ }^{\circledR}$, and many others that are in different stages of clinical investigation (Bobo et al. 2016; Brigger et al. 2002; Egusquiaguirre et al. 2012).

The particle size of polymer carriers is one of the key parameters that govern the bio-distribution of the product, and is also critical for bioavailability of drugs (Banerjee et al. 2016; Kumar et al. 2015; Yin; Win and Feng 2005). Therefore, a great effort has been invested in learning to control the fabrication process, mainly of degradable polymer particles, to produce particles with distinct and defined size. Amongst the most commonly used biocompatible polymers 
for drug delivery, are the PLGA polyesters. One of their great advantages is their predicted degradation rate that can be tuned by the ratio of the lactic and glycolic monomers and their molecular weight (Bret et al. 2011). PEG is another widely used polyester that is mainly utilized to evade the immune system and enhance blood circulation time (He et al. 2010; Kulkarni and Feng 2013). These two polymers are well-studied and are approved for use in various drug formulations by the Food and Drug Administration and the European Medicines Agency (Tabatabaei Mirakabad et al. 2014; Xie and Smith 2010).

Although well studied, microfluidic technologies for polymer particle fabrication have yet comprehensively adapted by the pharmaceutical industry, and particle synthesis still mostly performed in classical batch techniques, even prior to scaling up. One of the reasons for the low implementation of the technology is the limited number of simple and robust protocols for production of well-defined particles. In fact, the requirement to use different chip designs for nanoparticles versus microparticles' fabrication is often a hurdle for adaptation the technology. Two of the main methods for fabricating polymer particles using microfluidics, are the droplet generation and the solvent displacement methods for micron and nano sized carriers, respectively (Christopher and Anna 2007; Mosqueira et al. 2000; Prudhomme and Howard 2013; Quintanar-Guerrero et al. 1998).

Microfluidic devices for droplets generation are used for micron scales are based on combining two or more streams of immiscible fluids (Link et al. 2004). Focused-Flow chip design consists of a cross junction, where the drug and polymer organic solution dispersed phase enter through a central channel, and are squeezed at the orifice by a continuous aqueous phase. The laminar flow $(\operatorname{Re}<100)$ (Capretto et al. 2011) through the orifice enables a controlled droplets break-up that is required for yielding monodisperse microemulsions (Fig. 1A). For oil (O)-in water (W) systems that are commonly used for drug encapsulation, the $\mathrm{W}$ continuous phase imposes shear force that tears discontinues phase $\mathrm{O}$ into a stream of micron-sized monodispersed $\mathrm{O} / \mathrm{W}$ droplets. The droplets generally show much higher precision compared with those obtained by conventional methods.

Unlike the droplet generation method which is being used for producing micro-size particles, nano- and sub-micron particles are formed by rapid mixing and nanoprecipitation using the solvent displacement method and amphiphilic polymers (Beck-Broichsitter et al. 2010; Belliveau et al. 2012a; Makgwane and Ray 2014). In this method, the fluid stream containing the polymer precursor water miscible solution is squeezed into a narrow stream by two parallel aqueous streams with higher flow rates along the central channel, enabling rapid mixing through a spontaneous diffusion process (Fig. 1b). Indeed, successful examples of either nanoparticles' formulations in staggered herringbone micromixer
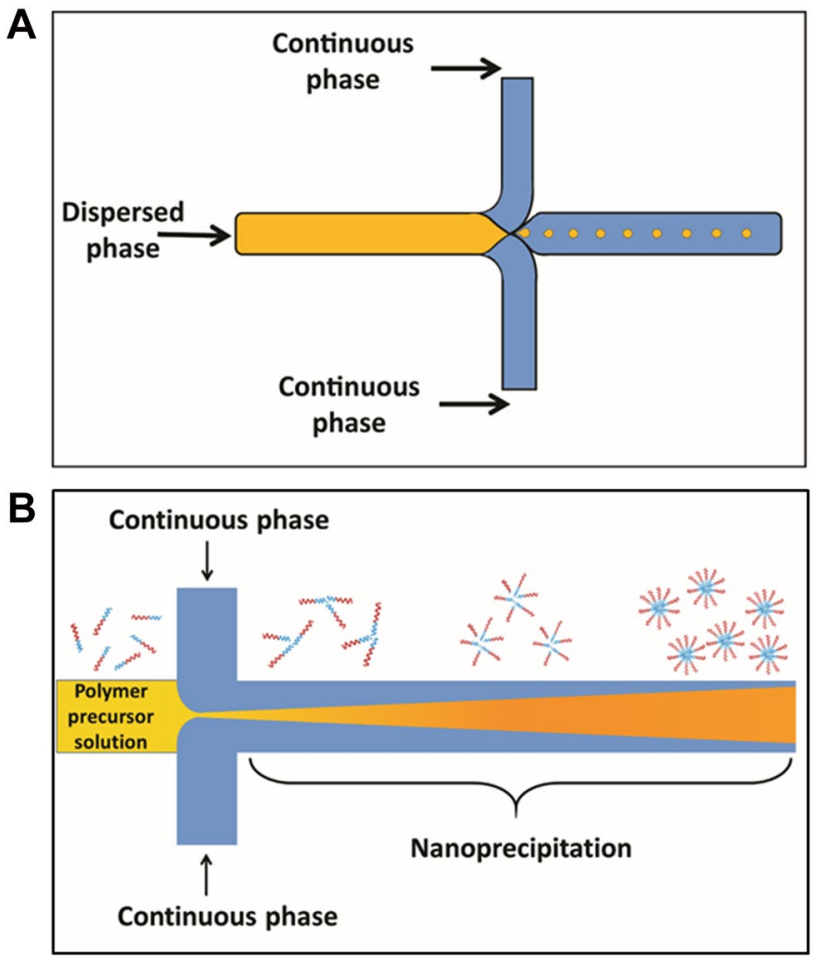

Fig. 1 Schematic illustration of junction in focused-flow geometry chip design for particles' synthesis. Magnified view of droplet generation and solvent displacement methods. a Schematic illustration of enlarged junction, controlled break-up droplet at the orifice. The flow through the orifice enables a controlled droplets break-up, which is required for yielding monodisperse micro-emulsions. b Polymer stream is squeezed into a narrow stream flow parallel each other to two adjacent aqueous streams flowing at higher flow rates along the central channel, enables precise nanoprecipitation through the rapid mixing and spontaneous diffusion process

(SHM) chips (Belliveau et al. 2012b; Maeki et al. 2015) or microparticles' formulations in droplet microfluidic devices can be found (Duncanson et al. 2012); however, very little, if any, studies show the fabrication of wide size range, from nanoparticles to microparticles in a single device.

The goal of our current study was to identify conditions and formulations in which polymeric nanoparticles and microparticles could be easily obtained using a single microfluidic design. Using a simple droplet generator design, we were able to systematically modify the polymer particle size and produce a well-controlled and homogenous formulation ranging from nanometers to microns. By changing either phases flow rates, polymer concentration, solvents miscibility or surfactants type, we can control and optimize the size of the particles while decreasing their polydispersity index, even without modifying the design of the chip. We prove that the choice of polymers and solvents is the main key factors in the production of polymeric particles using microfluidic devices. Changing the solvents miscibility and polymer composition affects interaction between the continuous 
and dispersive phases, and alter the synthesis method from droplet break-up to rapid mixing method thus producing submicron polymeric particles using the same chip design without any extra expenses. The narrow width of the focused stream flow in the rapid mixing method enables a slow and spontaneous diffusion of the aqueous and dispersive phases. This allows creating small polymeric nucleation spots that aggregate to form homogenous nanoparticles. Our work provides detailed methodological procedures for the formation of particles with varying sizes that can be used for more precise and simple synthesis of drug delivery systems.

\section{Materials and methods}

\section{Microfluidic system and chip}

Microfluidic system purchased from Micronit Microtechnologies (Netherland, Enschede). The chip is made of durable borosilicate glass and the fluidic slide is made of polypropylene. Chip dimensions are $45 \mathrm{~mm} \times 15 \mathrm{~mm}$, channel width and depth are 100 and $20 \mu \mathrm{m}$, respectively. All experiments performed using the flow-focusing design (Fig. 1).

\section{Droplets generation for the preparation of PLGA microparticles}

PLGA 50:50 (M.W. 40,000-75,000, Sigma-Aldrich) powder was dissolved in a water immiscible organic solvent, either dichloromethane (DCM) or toluene. This polymer mixture was emulsified in two aqueous solutions containing varying concentration of Sodium Deodecyl Sulfate (SDS, Sigma-Aldrich) and Polyvinyl Alcohol (PVA M.W. 67,000, Sigma-Aldrich) as emulsifying agents. The solutions stirred for $6 \mathrm{~h}$ to ensure complete solvent evaporation. The particles were washed three times with double distilled water (DDW), centrifuge in $800 \mathrm{~g}$ for $2 \mathrm{~min}$, and re-suspend with $2 \%$ mannitol solution. To prepare solidified particles, the washed particles' solution was frozen overnight in $-80{ }^{\circ} \mathrm{C}$ and lyophilized (Freezone 6 plus, Labconco, US) to produce a fine powder of particles that was stored at $-20{ }^{\circ} \mathrm{C}$.

\section{Solvent displacement for the preparation of PLGA-PEG nanoparticles}

Unlike the droplet synthesis, in the solvent displacement method, the polymer has to be dissolve in a water miscible organic solvent. In short, PLGA-PEG 10\% diblock (Boehringer Ingelheim) dissolved in acetonitrile (ACN) mixed with the aqueous phase. Upon dilution of the organic phase in the aqueous phase, the PLGA blocks, which are poorly soluble in water, spontaneously form particles' nucleation spots. The particles assemble until stabilization and the solutions stirred to verify complete solvent evaporation. To prepare solidified particles the collected sample was ultra-centrifuged at 80,000 g (TST28 rotor, Beckman's polyallomer centrifuge tubes), re-suspend with $2 \%$ mannitol solution, freeze at $-80{ }^{\circ} \mathrm{C}$ and lyophilized to produce a fine powder of particles that was stored at $-20^{\circ} \mathrm{C}$.

\section{Particle characterization}

The particles' samples examined and imaged using a scanning electron microscope (SEM) and a transmission electron microscope (TEM). For SEM (FEI Quanta 200 microscope) analysis, a small amount of the samples spread on a conductive adhesive carbon tape attached to an SEM grid. A thin film of Pd/Au coating sputtered onto the sample (SC7620 Spatter coater, UK). For TEM (Jeol, TEM 1400Plus, Japan, with charge-coupled camera-Gatan Orius SC600) analysis; 5 of particles' sample placed on a glow-discharged carbon coated 300 mesh copper TEM grid (Ted Pella, Inc). After blotting, the samples were negatively stained with $5 \mu 12 \%$ aqueous solution of uranyl acetate, and air-dried. Particle size was first evaluated by image analysis of TEM measurements using ImageJ program (https://imagej.nih.gov/ij/). Mean diameter of particles calculated based on the measurements of 30 randomly chosen particles. Size and charge analyses for nanoparticles performed using Dynamic Light Scattering (DLS)/Zeta sizer nanoseries (Malvern Instruments, UK) at $25^{\circ} \mathrm{C}$ in DTS1070 disposable capillary cells. $1 \mathrm{ml}$ of each sample was injected to measure the size and the zeta potential.

\section{Surface tension measurement}

The du Noüy ring method was applied to measure the surface tension of the PVA and SDS supplemented solution using tensiometer (KRUSS, K20 solid QC tensiometer, Germany) and platinum ring at room temperature. SDS and PVA solutions were prepared in $0.5,1$, and $2 \%$ concentrations. Prior to each measurement, the ring was rinsed with deionized water and sterilized under flame. $20 \mathrm{ml}$ of each surfactant solution was adequately shaken, poured into a glass flask, and measured. Measurements were repeated five times to represent the force needs to be applied for film tearing correctly.

\section{Statistics}

The experiments were performed with $n=3-4$. All data measurements represented as mean \pm standard deviation (SD). To identify statistically significant differences student's $t$ test used to compare two groups. One-way ANOVA followed by Tukey's test for post-test comparisons used when more than two groups compared. Probability values

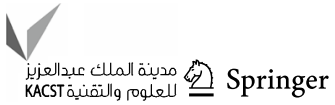


of $p<0.05$ and $p<0.01$ considered significant. ${ }^{*} p<0.05$; $* * p<0.01$.

\section{Results}

\section{Effect of flow rate on particle size}

Using a microfluidic platform, we were able to gently control the flow rate of the two joined phases with an electrical syringe pumps. Change in flow rate of the two immiscible fluids affected the size of the droplets to either increase or decrease their size. During the fabrication process, the dispersive phase rate remained constant with $0.05 \mathrm{ml} / \mathrm{min}$, while the continuous flow rate was set to either $0.4,0.5,0.6$, 0.8 , and $1 \mathrm{ml} / \mathrm{min}$. In these flow rate conditions, the average particle diameter decreased from $52 \pm 4,40 \pm 2,30 \pm 6$, $22 \pm 4$, and $6 \pm 2 \mu \mathrm{m}$, respectively (Fig. 2).

With respect to nanoparticles' synthesis, similar to the droplet generation process, the dispersed phase polymer concentration and flow rate were constant $(0.05 \mathrm{ml} / \mathrm{min})$, while the continuous flow rate was changed, leading to reduced particles' diameter. The effect of varying flow rates on the nanoparticles' diameter can be seen in Fig. 3 . All nanoparticles were prepared at $20 \mathrm{mg} / \mathrm{ml}$ concentration of PEG-PLGA and as we increased the flow rate of the continuous phase from 0.3 to 0.5 and $0.7 \mathrm{ml} / \mathrm{min}$ particles' average diameter decreased to $500 \pm 37,208 \pm 36$, and $40 \pm 10 \mathrm{~nm}$, respectively. Nanoparticles with continuous flow rate ratio of $14(0.7 / 0.05)$ yielded a smaller diameter compared with other low flow rate ratios.

\section{Effect of stabilizing agents on particle size}

Depending on the specific polymer solution, surfactants may be required to reduce the interfacial tension between the aqueous and organic phases and stabilize the dispersion. To determine the effect of surfactant on droplet size, PVA and SDS with elevated concentrations of $0.5,1$, and $2 \% \mathrm{wt} / \mathrm{vol}$ were used in the continuous phase using the droplet generation method. The flow rate of the system remained fixed in all experiments $(0.6 \mathrm{ml} / \mathrm{min}$ for the continuous phase $0.005 \mathrm{ml} / \mathrm{min}$ of the dispersed solution). In these conditions, elevated concentrations of SDS decreased the size of droplet diameter as follow: $30 \pm 5$, $22 \pm 5$, and $8 \pm 3 \mu \mathrm{m}$, respectively. The resulted diameter particles of the PVA solutions were $64 \pm 3,34 \pm 5$, and $14 \pm 4 \mu \mathrm{m}$ with elevated concentration, respectively (Fig. 4).

To examine the effect of surfactant concentration on nanoparticles' size, we prepared four continuous phase solutions with elevated percentage of PVA while maintaining all others parameters fixed throughout the experiment. The results summarized in Fig. 5. It can be seen that the nanoparticles' size has decreased from 186 to $132 \mathrm{~nm}$ with the increase of PVA concentration from 0.5 to $2 \% \mathrm{wt} / \mathrm{vol}$.
Fig. 2 Highly uniform PLGA microparticles are fabricated by droplet generation method. SEM images of PLGA particles fabricated at focused flow chip design. a Graph and table that summarizes particle diameter, continuous flow rates of 0.4 , $0.5,0.6,0.8$ and $1 \mathrm{ml} / \mathrm{min}$ with constant dispersed flow rate of $0.005 \mathrm{ml} / \mathrm{min}$. b Particles with mean diameter of $40 \mathrm{um}$. c Particles with mean diameter of $20 \mu \mathrm{m} . * p<0.05 ; * * p<0.01$

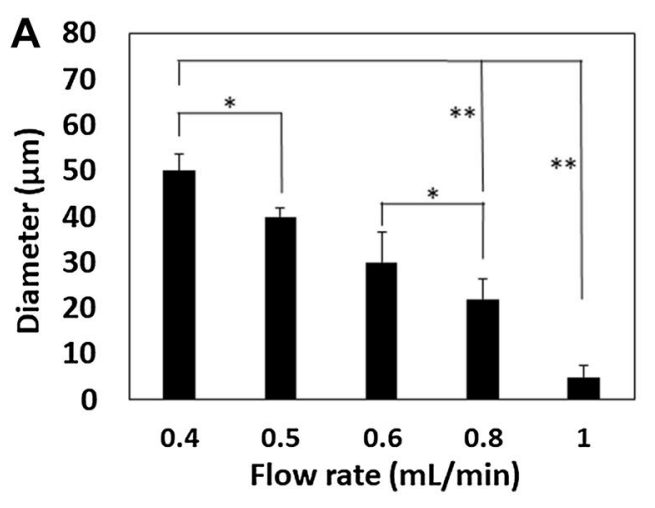

\begin{tabular}{|c|c|}
\hline $\begin{array}{c}\text { Continuous } \\
\text { phase flow rate } \\
(\mathrm{mL} / \mathrm{min})\end{array}$ & $\begin{array}{c}\text { Average } \\
\text { Diameter } \pm \text { SD } \\
(\boldsymbol{\mu})\end{array}$ \\
\hline 0.4 & $53 \pm 4$ \\
\hline 0.5 & $40 \pm 2$ \\
\hline 0.6 & $31 \pm 6$ \\
\hline 0.8 & $20 \pm 4$ \\
\hline 1 & $6 \pm 2$ \\
\hline
\end{tabular}
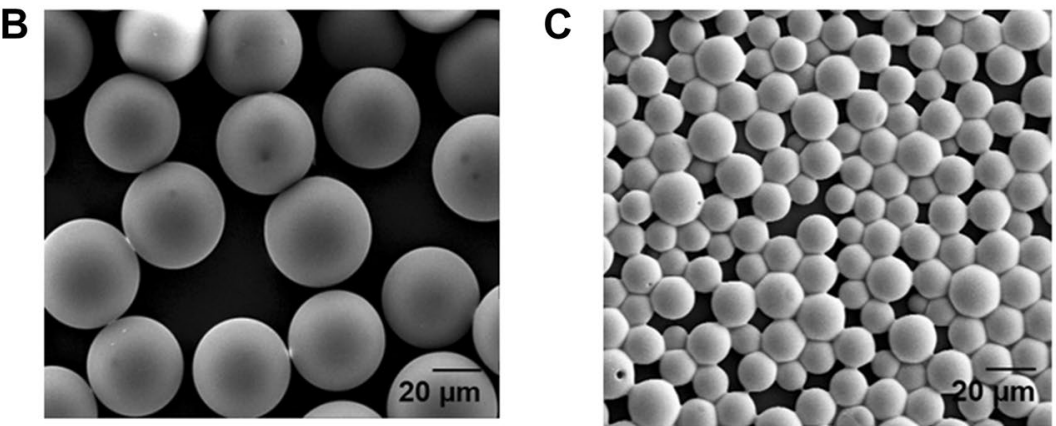
Fig. 3 Diameter of PEG-PLGA nanoparticles can be controlled by gently varying continuous phase flow rates. Images of PEG-PLGA 1\% solution particles fabricated at focused flow chip design. Continuous rates of $0.3,0.5$ and $0.7 \mathrm{ml} / \mathrm{min}$ with constant dispersed flow rate of $0.005 \mathrm{ml} / \mathrm{min}$. a Nanoparticles' homogeneity summarized graph obtained in varying flow rates by rapid mixing solvent displacement method of $1 \%$ PEG-PLGA solution, b size measurement obtained by dynamic light scattering (DLS). $\mathbf{c}, \mathbf{d}$ TEM and SEM images of PEG-PLGA particles, respectively. ${ }^{*} p<0.05 ; * * p<0.01$
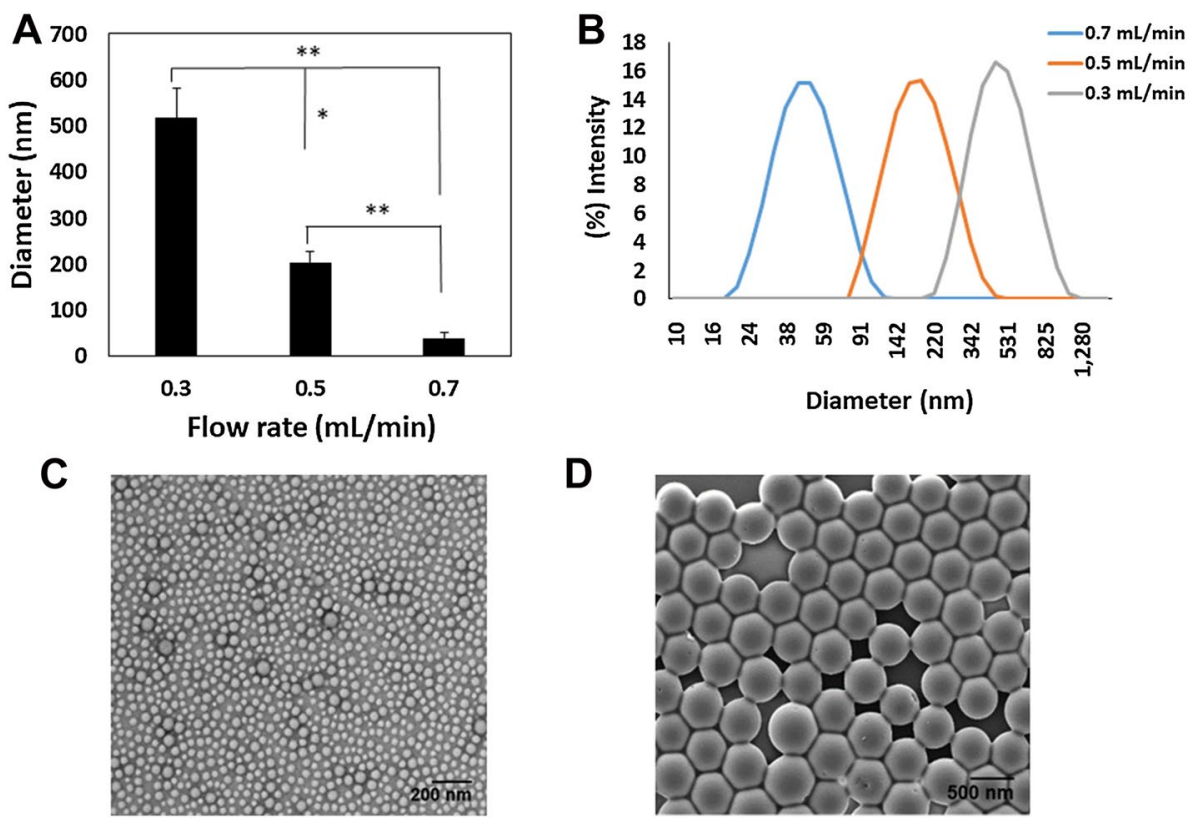

D

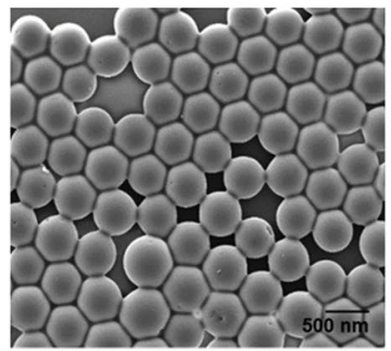

Fig. 4 Effect of surfactant type and concentration on microparticles fabricated by droplet generation method. SEM images of PLGA particles fabricated by focused flow chip design. a Influence of surfactant concentration and type on particles' diameter using PVA and SDS solutions. Continuous phase flow rates of $0.6 \mathrm{ml} /$ min and dispersed flow rate of $0.005 \mathrm{ml} / \mathrm{min}$. b Particles fabricated with SDS $0.5 \%$ and c particles fabricated with PVA $1 \%$. Each sample diameters were calculated based on 30 randomly chosen particles using ImageJ. $* p<0.05, N S$ not significant

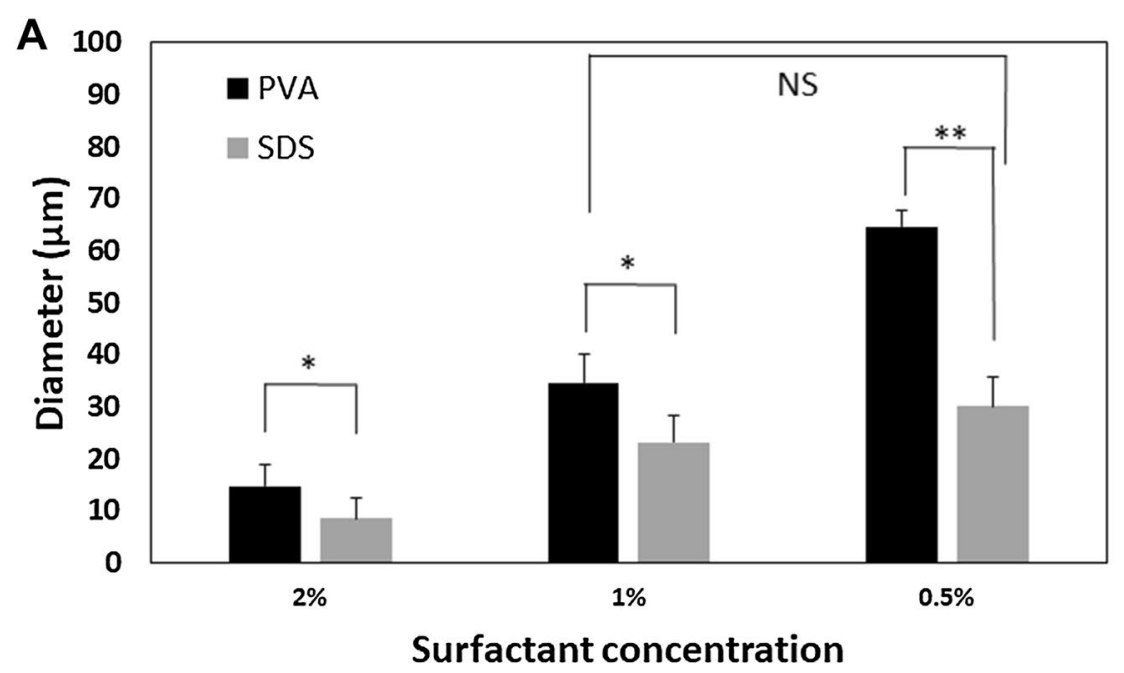

B

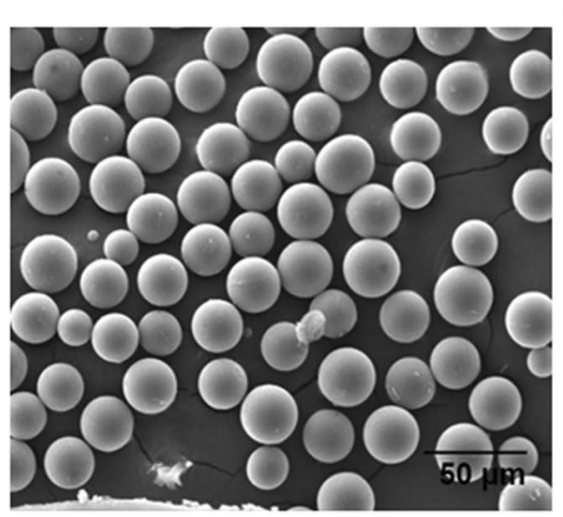

c

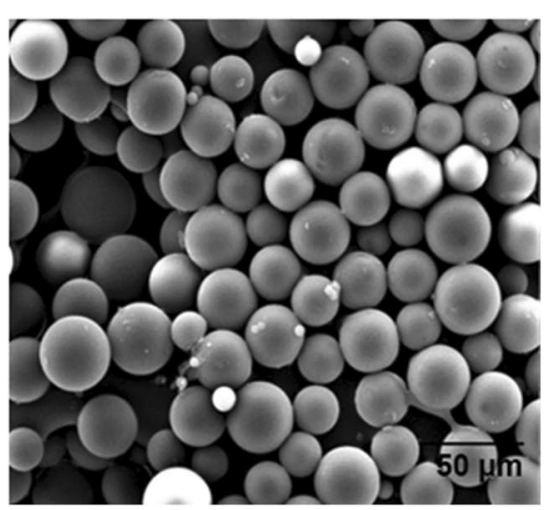




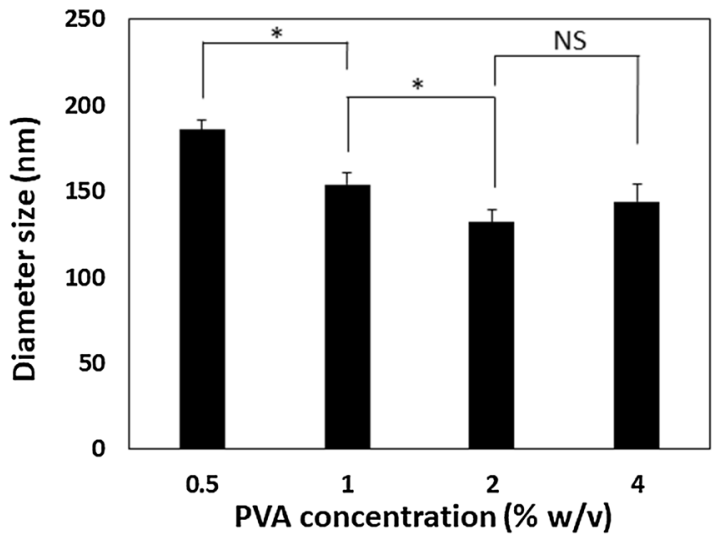

\begin{tabular}{|c|c|c|}
\hline \% PVA w/v & $\begin{array}{c}\text { Mean diameters } \\
\pm \text { SD (nm) }\end{array}$ & Polydispersity \\
\hline 0.5 & $186 \pm 6$ & 0.067 \\
\hline 1 & $153 \pm 7$ & 0.081 \\
\hline 2 & $132 \pm 7$ & 0.046 \\
\hline 4 & $144 \pm 10$ & 0.1 \\
\hline
\end{tabular}

Fig. 5 Influence of surfactant concentration on nanoparticles' mean diameter and size distribution. Diameter of nanoparticles can be controlled by varying surfactant concentration $(\% \mathrm{w} / \mathrm{v})$ in the continuous phase. ${ }^{*} p<0.05$. NS not significant

\section{Effect of polymer concentration on particle size}

Another main parameter affecting nanoparticles' size and stability is the polymer concentration. Modifying this factor provides an additional degree of freedom, allowing for the optimization of the formulation properties without changing other intrinsic factors. Increasing the concentration of the polymer from 5 to $40 \mathrm{mg} / \mathrm{ml}$ has substantially increased the average particles' size from 50 to $200 \mathrm{~nm}$ (Fig. 6).

\section{Discussion}

The field of microfluidics for nanoparticle synthesis has been widely developed in the past two decades, though there is still a great need of fundamental research and attention to develop new fabrication protocols using this powerful platform. In this work, we demonstrate the potential benefit of using microfluidics for polymeric particles' fabrication for drug delivery, and the ability to control variety of parameters to fine-tune particles' size over a wide size range while using a single chip design (Table 1 summarizes experiments varying parameters).

Particles' size of drug carriers is a major deterministic factor in the pattern of the body biodistribution and preparations solubility (Alexis et al. 2008; He et al. 2010; Kulkarni and Feng 2013; Lvov et al. 2011; Yin; Win and Feng 2005). The ultimate goal in drug delivery is to reduce off-target effects by improving the targeting of the drug to the desired tissue. Therefore, there is a major need to develop ways to better and tightly control the size of particles using a robust method. Along this aim, microfluidics may provide a very powerful method compared with batch preparation of particles such as extrusion, batch emulsification, and layer by layer. It can be seen in various nanoparticles' synthesis approaches-from nucleic acid and drug lipid nanoparticles'
Fig. 6 Effect of PEG-PLGA polymer concentration on the diameter and PDI of the nanoparticles using focused flow chip. a Nanoparticles' average size can be controlled by varying polymer concentration. $\mathbf{b}$ Size distribution measurement of polymeric nanoparticles obtained by DLS. ${ }^{*} p<0.05$; $* * p<0.01$

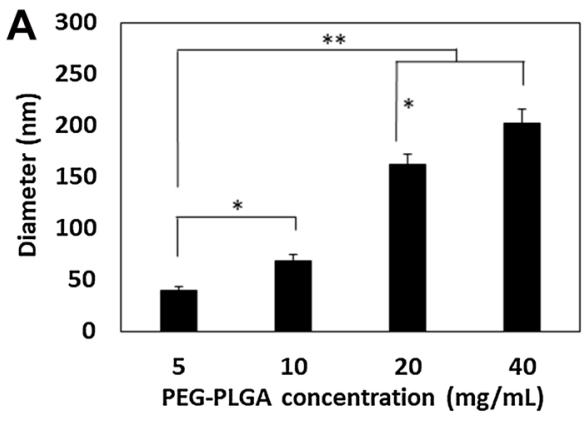

\begin{tabular}{|c|c|c|}
\hline $\begin{array}{c}\text { \% PEG-PLGA } \\
\text { w/v }\end{array}$ & $\begin{array}{c}\text { Mean } \\
\text { diameters } \pm \\
\text { SD (nm) }\end{array}$ & $\begin{array}{c}\text { Polydispersity } \\
\text { (PDI) }\end{array}$ \\
\hline 0.5 & $40 \pm 3$ & 0.083 \\
\hline 1 & $68 \pm 6$ & 0.091 \\
\hline 2 & $161 \pm 10$ & 0.1 \\
\hline 4 & $202 \pm 12$ & 0.096 \\
\hline
\end{tabular}

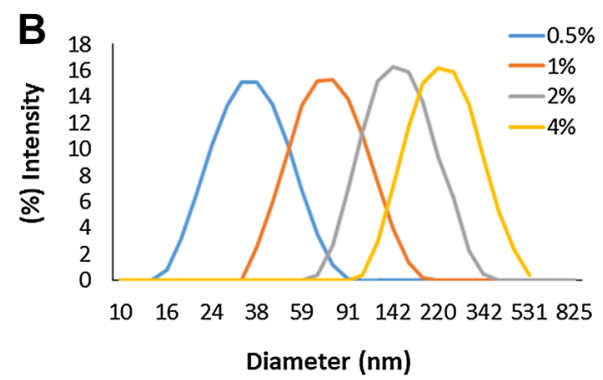


Table 1 Primary table that summarizes all fabrication parameters and methods for micro/nano-particles' synthesis

\begin{tabular}{|c|c|c|c|c|c|}
\hline \multirow{2}{*}{$\begin{array}{c}\begin{array}{c}\text { Synthesis } \\
\text { property }\end{array} \\
\begin{array}{c}\text { Tested } \\
\text { parameter }\end{array}\end{array}$} & \multicolumn{2}{|c|}{ Flow rate } & \multirow{2}{*}{ Surfactant type } & \multirow{2}{*}{$\begin{array}{l}\text { Polymer } \\
(\% \mathrm{w} / \mathrm{v})\end{array}$} & \multirow{2}{*}{$\begin{array}{l}\text { PVA } \\
(\% \mathrm{w} / \mathrm{v})\end{array}$} \\
\hline & Microparticles & Nanoparticles & & & \\
\hline Polymer type & PLGA (50:50) & PEG-PLGA $10 \%$ & PLGA (50:50) & PEG-PLGA $10 \%$ & PEG-PLGA $10 \%$ \\
\hline Organic phase & DCM & $\mathrm{ACN}$ & DCM & $\mathrm{ACN}$ & $\mathrm{ACN}$ \\
\hline Aqueous phase & \multicolumn{2}{|c|}{ 1\% PVA } & $\begin{array}{r}0.5,1,2 \% \\
\text { SDS or PVA }\end{array}$ & $1 \%$ PVA & $\begin{array}{c}0.5,1,2,4 \% \\
\text { PVA }\end{array}$ \\
\hline $\begin{array}{l}\text { Aqueous phase rate } \\
(\mathrm{mL} / \mathrm{min})\end{array}$ & $\begin{array}{c}0.4,0.5,0.6 \\
\quad 0.8,1\end{array}$ & $0.3,0.5,0.7$ & 0.6 & 0.5 & 0.5 \\
\hline $\begin{array}{l}\text { Dispersed phase rate } \\
\text { (mL/min) }\end{array}$ & \multicolumn{2}{|c|}{0.005} & 0.005 & 0.005 & 0.005 \\
\hline $\begin{array}{l}\text { Polymer concentration } \\
\qquad(\% \mathrm{w} / \mathrm{v})\end{array}$ & \multicolumn{2}{|c|}{$2 \%$} & $2 \%$ & $0.5,1,2,4 \%$ & $2 \%$ \\
\hline
\end{tabular}

Abbreviations: PLGA, poly(lactide-co-glycolide); PEG, polyethylene glycol; DCM, dichloromethane; ACN, acetonitrile; PVA, polyvinyl alcohol; SDS, sodium deodecyl sulfate systems that exhibit higher encapsulation efficiency and smaller diameter, to improved drug screening process and new hybrid metallic nanoparticles' synthesis (Belliveau et al. 2012a; Carugo et al. 2016; Khan et al. 2014; Vergaro et al. 2011; Zhang et al. 2015). As continuous flow rate increases in both droplet generation and solvent displacement methods, particles' size decreases.

The increase in the aqueous flow rate compared with the organic flow rate enables the decrease in droplets size and consequently in smaller polymeric particles. Accordingly, a decrease in the flow rate would lead to an increase in droplets size due to slow process of the droplets, cutting at the orifice region.

With respect to solvent displacement method, to ensure homogeneous nanoparticles' preparation, nucleation process must be done over a very short period of time (Abou-Hassan et al. 2009; Mora-Huertas et al. 2011; Di Pasquale et al. 2012). This principle can be enforced through the unique junction design of the microfluidic chip which allows the organic phase to be added slowly through the orifice to maintain a polymer concentration level that is lower than the critical concentration, at which new nucleation will be carried out. The overall nucleation process and parameters affecting it are being discussed at length in Chen et al., Murray et al., and Cozzoli et al. (Chen et al. 2014; Cozzoli et al. 2006; Heights 2000).

The ability to achieve a laminar flow at a microscopic level using microfluidics devices and to control the flow rate dictates the mixing time and consequently the diffusion process between the two joined phases. Karnik et al. have shown that increasing the continuous phase rate ensures faster mixing and decreases the mixing time. Using microfluidic device with flow focusing, they demonstrated the fabrication of PLGA nanoparticles with diameters range of 10-50 nm (Karnik et al. 2008). As demonstrated, we show that the more significant differences in flow rates are, the thinner the dispersed phase stream is at the outlet channel, with decreased nanoparticles' size. Although we present a fine-tuning ability to control and vary the flow rates of the two-joined phases, this process has its natural limit. When the dispersive $(\mathrm{O})$ phase flow rate is too slow, droplets are not produced due a back flow pressure of the aqueous (W) continues phase through the orifice.

Unlike the laminar flow scenario, a fast flow rate might cause a turbulent flow with significant reduction in the controlled droplets break-up process and heterogeneous mixing. This main parameter has a direct effect on mixing efficiency within microfluidic devices, particles' assembly, and, as a result, the particles' size. Our results are in agreement with other studies that showed the ability to achieve precise control over the size only with varying the flow rates (Fu et al. 2012; Luo et al. 2011; Zhao 2013).

Varying the concentration of the polymer and surfactant found to be a simple and efficient parameter that controls particles' size and formulation stability. With regard to nanoparticles' synthesis, as polymer concentration increases, particles' size increases as well. This phenomenon might be explained by the fact that increased polymer concentration leads to a higher viscosity of the solution. Increasing solution viscosity eventually decreases the diffusion rate, which increases the mixing time and, therefore, influences the particles' size (Budhian et al. 2007). This can also be shown by diffusion constant (Capretto et al. 2011). 
Surfactants are essential components in the production of long-living emulsions, as they stabilize the interphase between the aqueous and organic phases by reducing interfacial surface tension. Usually, small concentration of $0.5-4 \% \mathrm{wt} / \mathrm{vol}$ is sufficient to reduce the macroscopically coalescence due to spontaneous thermodynamic equilibrium stable state (Abdelwahed et al. 2006; Duncanson et al. 2012). We examined whether PVA and SDS affect particles' size diameter in the droplet generation method, or solely stabilize the fabricated dispersions compared with droplets without any surfactant. In both experiments, the average droplet diameter tends to decrease as the surfactant concentration increased, which may be explained by the higher availability of surfactant molecules overlaying the surface of the fabricated droplets (Lee et al. 1999), thus preventing spontaneous coalescence, results in the production of smaller droplets. When comparing the two surfactants, we showed that $0.5 \%$ SDS and $1 \%$ PVA yielded similar droplet size of $30 \mu \mathrm{m}$ on average, with a slight higher in diversity with PVA which might be explained by the increased viscosity of the PVA compared with that of the SDS solution (Lee et al. 1999; Vysloužil et al. 2014). In general, particles fabricated with PVA solutions showed larger diameters compared to those fabricated with SDS in the same concentration. To explain these differences, we measured the dynamic interfacial tensions of the surfactant solutions concentrations using the du Noüy ring method. The dynamic interfacial tensions measured for SDS solutions were: $35.5,34.8$, and 34 dyne/cm, $(0.5,1$, and $2 \%$, respectively), while PVA solution showed 49.8, 47.5, and 45.5 dyne/cm, respectively. The lower interfacial tensions obtain with SDS explains the generation and stabilization of smaller drops (Binks 2002; Tong et al. 2000). In addition, the fact that SDS is an anionic surfactant may contribute to electrical repulsion between the fabricated droplets. Such repulsion may contribute to the system thermodynamically stability by reducing droplets collision and coalescence. (Castelvetro et al. 2006; Tcholakova et al. 2004).

Importantly, it should be noted that the absence of stabilizing agent leads to failure in producing any particles due to droplet coalescence. These indicates that the main mechanism of stabilization was prevention of droplet generation rather than droplet generation and reduction of particles' size.

In the case of solvent displacement method, we found that higher concentration of PVA led to a reduction in particles' size up to a certain limit. These results can be explained by the reduction in interfacial tension as the concentration of PVA increases. Insufficient amount of stabilizer fails to stabilize the massive mass of the produced nanoparticles causing aggregation due to thermodynamically equilibrium stable state (Menon et al. 2012; Sharma et al. 2016). As a result, nanoparticle with higher diameter would be fabricate. An additional increase in PVA concentration to $4 \% \mathrm{wt} / \mathrm{vol}$ did not reduce particles' size, rather increased it to $144 \mathrm{~nm}$.
Although this observation was not found to be statistically significant, it can be explained by the increased viscosity of the continuous phase that reduces the ability of the dispersed phase to flow through the continuous solution with the flow rates set to this experiment. We show that PVA 2\% wt/vol concentration is sufficient to stabilize these nanoparticles at these fabrication conditions.

\section{Conclusion}

Two different methods, for micro- and nanoparticles' synthesis, were discussed. Both methods were based and demonstrated using single microfluidic chip design. Previously, most of micro-and nanoparticles' production based on 'batch' synthesis methods. The leading trend nowadays is changing towards fluid flow at the microscopic scale for particles' production that characterizes simple reproducibility and narrow size distribution.

Although it is imperative to develop a reliable method for producing monodisperse drug carriers, the use of microfluidic devices is not without limitations. Assembling of the flow system and optimization using a chip with specific designs requires special tools, training, and long a fabrication process. In addition, this unique channel geometry either may clog, by unstable movements of worktop platform or by particles' debris, and it requires specialized cleaning procedures to overcome it.

Future research work should focus on the development of new fabrication techniques and a more precise control over particle morphology, which is a main determinant for innovative formulation and applications. With regard to nanoparticles' production, mixing efficiency can be increased by incorporating in the central channel mixing steps, which creates additional perturbations that increase the surface area encounter of the phases and decrease the mixing time. In comparison with traditional methods, we clearly show the promise of microfluidics technologies integration into the pharmaceutical industries, especially in the formulation optimization phase that may replace classical batch synthesis methods in the near future. Moreover, this method can offer a powerful tool for production of micro- and nanoencapsulated drugs "on demand" for personalized medicine.

Acknowledgements We gratefully acknowledge the support from the Israel Science Foundation; Marie Curie CIG, and The Israeli Ministry of Science and Technology. This project has received funding from the European Research Council (ERC) under the European Union's Horizon 2020 research and innovation programme (grant agreement No 756762) and Kamin fund from the Israeli Innovation Authority. We wish to thank Dr. Eduard Berenstein from the Core Research Facility of the Hebrew University for his help in the TEM and SEM studies. We also would like to thank Dr. Vitaly Gutkin from the Nano-center of the Hebrew University for his help in the SEM studies. 


\section{Compliance with ethical standards}

Conflict of interest On behalf of all authors, the corresponding author states that there is no conflict of interest.

Open Access This article is distributed under the terms of the Creative Commons Attribution 4.0 International License (http://creativeco mmons.org/licenses/by/4.0/), which permits unrestricted use, distribution, and reproduction in any medium, provided you give appropriate credit to the original author(s) and the source, provide a link to the Creative Commons license, and indicate if changes were made.

\section{References}

Abdelwahed W, Degobert G, Stainmesse S, Fessi H (2006) Freezedrying of nanoparticles: formulation, process and storage considerations. Adv Drug Deliv Rev 58(15):1688-1713

Abou-Hassan A, Sandre O, Neveu S, Valérie, Cabuil (2009) Synthesis of goethite by separation of the nucleation and growth processes of ferrihydrite nanoparticles using microfluidics. Angew Chem 48:2342-2345

Alexis F, Pridgen E, Linda K, Molnar, Farokhzad OC (2008) Factors affecting the clearance and biodistribution of polymeric nanoparticles. Mol Pharm 5(4):505-515

Banerjee A et al (2016) Role of nanoparticle size, shape and surface chemistry in oral drug delivery. J Control Release 238:176-185. https://doi.org/10.1016/j.jconrel.2016.07.051

Beck-Broichsitter M et al (2010) Preparation of nanoparticles by solvent displacement for drug delivery: a shift in the 'ouzo Region' upon drug loading. Eur J Pharm Sci 41(2): 244-253. http://www. ncbi.nlm.nih.gov/pubmed/20600881. Accessed 16 Sept 2015

Belliveau NM et al (2012a) Microfluidic synthesis of highly potent limit-size lipid nanoparticles for in vivo delivery of siRNA. Mol Therapy Nucleic Acids 1:e37. http://linkinghub.elsevier.com/retri eve/pii/S2162253116300932. Accessed 7 Sept 2017

Belliveau NM et al (2012b) Microfluidic synthesis of highly potent limit-size lipid nanoparticles for in vivo delivery of siRNA. Mol Therapy Nucleic Acids 1:1-9

Binks BP (2002) Particles as surfactants—similarities and differences. Curr Opin Colloid Interf Sci 7(1-2):21-41

Bobo D et al (2016) Nanoparticle-based medicines: a review of FDA-approved materials and clinical trials to date. Pharm Res 33(10):2373-2387

Bret UD, Nair S, Lakshmi, Cato TL (2011) Biomedical applications of biodegradable polymers. J Polym Sci Part B: Polym Phys 3(49):832-864

Brigger I, Dubernet C, Couvreur P (2002) Nanoparticles in cancer therapy and diagnosis. Adv Drug Deliv Rev 54(5):631-651

Budhian A, Siegel SJ, Winey KI (2007) Haloperidol-loaded PLGA nanoparticles: systematic study of particle size and drug content. Int J Pharm 336(2):367-375

Capretto L, Cheng W, Hill M, Zhang X (2011) Micromixing within microfluidic devices. In: Lin B (eds) Microfluidics. Topics in Current Chemistry, vol 304, pp 27-68. Springer, Berlin

Carugo D et al (2016) Liposome production by microfluidics: potential and limiting factors. Sci Rep 6(1):25876. http://www.nature.com/ articles/srep25876

Castelvetro V, Vita CD, Giannini G, Giaiacopi S (2006) Role of anionic and nonionic surfactants on the control of particle size and latex colloidal stability in the seeded emulsion polymerization of butyl methacrylate. J Appl Polym Sci 102(4):3084-3094
Chen L-Y et al (2014) Rapid control of phase growth by nanoparticles. Nat Commun 5:1-9. http://www.nature.com/doifinder/10.1038/ ncomms 4879

Christopher GF, Anna SL (2007) Microfluidic methods for generating continuous droplet streams. J Phys D Appl Phys 40(19):R319R336. http://stacks.iop.org/0022-3727/40/i=19/a=R01 ?key=cross ref.d7a86ce1ad957acd3e27d1104e98069f

Cozzoli P, Davide T, Pellegrino, Manna L (2006) Synthesis, properties and perspectives of hybrid nanocrystal structures. Chem Soc Rev 35(11):1195. http://xlink.rsc.org/?DOI=b517790c

Di Pasquale N, Marchisio DL, Barresi AA (2012) Model validation for precipitation in solvent-displacement processes. Chem Eng Sci 84:671-683. https://doi.org/10.1016/j.ces.2012.08.043

Duncanson WJ et al (2012) Microfluidic synthesis of advanced microparticles for encapsulation and controlled release. Lab Chip 12(12):2135-2145. http://www.ncbi.nlm.nih.gov/pubmed/22510 961

Egusquiaguirre SP, Igartua M, Hernández RM, Pedraz JL (2012) Nanoparticle delivery systems for cancer therapy: advances in clinical and preclinical research. Clin Transl Oncol 14(2):83-93

Fu T, Wu Y, Ma Y, Li HZ (2012) Droplet formation and breakup dynamics in microfluidic flow-focusing devices: from dripping to jetting. Chem Eng Sci 84:207-217. http://www.sciencedirect.com/ science/article/pii/S0009250912005441. Accessed 23 Nov 2014

He C et al (2010) Effects of particle size and surface charge on cellular uptake and biodistribution of polymeric nanoparticles. Biomaterials 31(13):3657-3666. https://doi.org/10.1016/j.biomateria 1s.2010.01.065

Heights Y (2000) Synthesis and characterization of monodisperse nanocrystals and close-packed nanocrystal assemblies. Annu Rev Mater Sci 30:545-610

Jain S, Hirst DG, O'Sullivan JM (2012) Gold nanoparticles as novel agents for cancer therapy.Br J Radiol 85(1010):101-113. http:// www.birpublications.org/doi/10.1259/bjr/59448833

Karnik R et al (2008) Microfluidic platform for controlled synthesis of polymeric nanoparticles.Nano Lett 8(9):2906-2912. http:// www.ncbi.nlm.nih.gov/pubmed/18656990

Khadka P et al (2014) Pharmaceutical particle technologies: an approach to improve drug solubility, dissolution and bioavailability. Asian J Pharm Sci 9(6):304-316. https://doi. org/10.1016/j.ajps.2014.05.005

Khan IU, Serra CA, Anton N, Vandamme TF (2014) Production of nanoparticle drug delivery systems with microfluidics tools. Exp Opin Drug Delivery 12(4):1-16. http://www.ncbi.nlm.nih. gov/pubmed/25345543

Kulkarni SA, Feng SS (2013) Effects of particle size and surface modification on cellular uptake and biodistribution of polymeric nanoparticles for drug delivery. Pharm Res 30(10):2512-2522. http://link.springer.com/10.1007/s11095-012-0958-3

Kumar S et al (2015) Shape and size-dependent immune response to antigen-carrying nanoparticles. J Control Release 220:141-148. https://doi.org/10.1016/j.jconrel.2015.09.069

Lee SC, Oh JT, Jang MH, Chung SI (1999) Quantitative analysis of polyvinyl alcohol on the surface of poly(D,L-Lactide-Co-Glycolide) microparticles prepared by solvent evaporation method: effect of particle size and PVA concentration. J Control Release 59(2):123-132

Link DR, Anna SL, Weitz DA, Stone HA (2004) Geometrically mediated breakup of drops in microfluidic devices. Phys Rev Lett 92(5):54503. https://link.aps.org/doi/10.1103/PhysRevLet t.92.054503

Luo $\mathrm{G}$ et al (2011) Controllable preparation of particles with microfluidics. Particuology 9(6):545-558. http://www.sciencedirect. com/science/article/pii/S1674200111001325. Accessed 4 Nov 2014 
Lvov YM, Pattekari P, Zhang X, Torchilin V (2011) Converting poorly soluble materials into stable aqueous nanocolloids. Langmuir 27(3):1212-1217

Maeki M et al (2015) A strategy for synthesis of lipid nanoparticles using microfluidic devices with a mixer structure. RSC Adv 5(57):46181-46185. http://xlink.rsc.org/?DOI=C5RA04690D

Makgwane PR, Ray SS (2014) Synthesis of nanomaterials by continuous-flow microfluidics: a review. J Nanosci Nanotechnol 14(2):1338-63. http://openurl.ingenta.com/content/xref?genre $=$ article $\&$ issn $=1533-4880 \&$ volume $=14 \&$ issue $=2 \&$ spage $=1338$

Menon JU et al (2012) Effects of surfactants on the properties of PLGA nanoparticles. J Biomed Mater Res Part A 100(8):1998-2005. http://www.ncbi.nlm.nih.gov/pubmed/22566409. Accessed 3 Aug 2015

Mora-Huertas CE, Fessi H, Elaissari A (2011) Influence of process and formulation parameters on the formation of submicron particles by solvent displacement and emulsification-diffusion methods: critical comparison. Adv Coll Interface Sci 163(2):90-122. https ://doi.org/10.1016/j.cis.2011.02.005

Mosqueira VCF et al (2000) Poly(D,L-Lactide) nanocapsules prepared by a solvent displacement process: influence of the composition on physicochemical and structural properties. J Pharm Sci 89(5):614-626

Niu Y et al (2017) Smart multifunctional polyurethane microcapsules for the quick release of anticancer drugs in BGC 823 and HeLa tumor cells. J Mater Chem B 5:9477-9481. http://xlink.rsc. org/?DOI=C7TB02570J

Novotný J, Foret F (2017) Fluid manipulation on the micro-scale: basics of fluid behavior in microfluidics. J Sep Sci 40(1):383-394

Parveen S, Sahoo SK (2008) Polymeric nanoparticles for cancer therapy. J Drug Target 16(2):108-123

Prudhomme RK, Howard AS (2013) NIH Public Access 28(37)

Quintanar-Guerrero D, Allémann E, Fessi H, Doelker E (1998) Preparation techniques and mechanisms of formation of biodegradable nanoparticles from preformed polymers. Drug Dev Ind Pharm 24(12):1113-1128. http://www.tandfonline.com/doi/ full/10.3109/03639049809108571

Sharma N, Madan P, Lin S (2016) Effect of process and formulation variables on the preparation of parenteral paclitaxel-loaded biodegradable polymeric nanoparticles: a co-surfactant study. Asian J Pharm Sci 11(3):404-416. https://doi.org/10.1016/j. ajps.2015.09.004

Squires TM (2005) Microfluidics fluid physics at the Nanoliter scale. Rev Mod Phys 77(3):977
Tabatabaei M, Fatemeh S et al (2014) PLGA-based nanoparticles as cancer drug delivery systems. Asian Pacif J Cancer Prev 15(2):517-35. http://koreascience.or.kr/journal/view. jsp?kj=POCPA9\&py $=2014 \& v n c=v 15 n 2 \& s p=517$

Tcholakova S, Denkov ND, Banner T (2004) Role of surfactant type and concentration for the mean drop size during emulsification in turbulent flow. Langmuir 20(18):7444-7458

Tong J, Nakajima M, Nabetani H, Kikuchi Y (2000) Surfactant effect on production of monodispersed microspheres by microchannel emulsification method. J Surfactants Deterg 3(3):285-293

Valencia PM, Omid C, Farokhzad R, Karnik, Langer R (2012) Microfluidic technologies for accelerating the clinical translation of nanoparticles. Nat Nanotechnol 7(10):623-629. https://doi. org/10.1038/nnano.2012.168

Vergaro V et al (2011) Drug-loaded polyelectrolyte microcapsules for sustained targeting of cancer cells. Adv Drug Deliv Rev 63(9):847-864

Vysloužil J et al (2014) Influence of different formulations and process parameters during the preparation of drug-loaded PLGA microspheres evaluated by multivariate data analysis. Acta Pharm 64(4):403-417. http://www.degruyter.com/view/j/acph.2014.64. issue-4/acph-2014-0032/acph-2014-0032.xml

Win YK, Feng SS (2005) Effects of particle size and surface coating on cellular uptake of polymeric nanoparticles for oral delivery of anticancer drugs. Biomaterials 26(15):2713-2722. http://linki nghub.elsevier.com/retrieve/pii/S0142961204006921

Xie H, Smith JW (2010) Fabrication of PLGA nanoparticles with a fluidic nanoprecipitation system. J Nanobiotechnol 8:18

Zhang Lu et al (2015) Microfluidic synthesis of hybrid nanoparticles with controlled lipid layers: understanding flexibility-regulated cell-nanoparticle interaction. ACS Nano 9(10):9912-9921

Zhao C-X (2013) Multiphase flow microfluidics for the production of single or multiple emulsions for drug delivery. Adv Drug Delivery Rev 65(11-12):1420-1446. http://www.sciencedirect.com/scien ce/article/pii/S0169409X13001476. Accessed 26 Sept 2014

Publisher's note Springer Nature remains neutral with regard to jurisdictional claims in published maps and institutional affiliations. 